\title{
High quality ion acceleration through the interaction of two matched counterpropagating transversely polarized Gaussian lasers with a flat foil target
}

\author{
Weijun Zhou, ${ }^{1}$ Xueren Hong, ${ }^{1,}{ }^{*}$ Baisong Xie, ${ }^{2}$ Yang Yang, ${ }^{1}$ Li Wang, ${ }^{1}$ \\ Jianmin Tian, ${ }^{1}$ Rongan Tang, ${ }^{1}$ and Wenshan Duan ${ }^{1}$ \\ ${ }^{1}$ Key Laboratory of Atomic and Molecular Physics \& Functional Materials of Gansu Province, \\ College of Physics and Electronic Engineering, Northwest Normal University, Lanzhou 730070, China \\ ${ }^{2}$ College of Nuclear Science and Technology, Beijing Normal University, Beijing 100875, China
}

(Received 29 August 2017; published 2 February 2018)

\begin{abstract}
In order to generate high quality ion beams through a relatively uniform radiation pressure acceleration (RPA) of a common flat foil, a new scheme is proposed to overcome the curve of the target while being radiated by a single transversely Gaussian laser. In this scheme, two matched counterpropagating transversely Gaussian laser pulses, a main pulse and an auxiliary pulse, impinge on the foil target at the meantime. It is found that in the two-dimensional (2D) particle-in-cell (PIC) simulation, by the restraint of the auxiliary laser, the curve of the foil can be effectively suppressed. As a result, a high quality monoenergetic ion beam is generated through an efficient RPA of the foil target. For example, two counterpropagating transversely circularly polarized Gaussian lasers with normalized amplitudes $a_{1}=120$ and $a_{2}=30$, respectively, impinge on the foil target at the meantime, a $1.3 \mathrm{GeV}$ monoenergetic proton beam with high collimation is obtained finally. Furthermore, the effects on the ions acceleration with different parameters of the auxiliary laser are also investigated.
\end{abstract}

DOI: 10.1103/PhysRevAccelBeams.21.021301

\section{INTRODUCTION}

The high quality ion acceleration through the interaction of the intense laser with plasma has been widely studied in recent years, which has many significant applications in diverse fields, such as low-cost tabletop accelerator [1], hadron therapy of oncological diseases [2] and the fast ignition in the inertial confinement fusion [3]. Compared with the traditional particle accelerators, the laser plasma accelerator has some advantages in the ultrahigh accelerating gradient, smaller size, and relatively lower cost [4]. For now, some acceleration mechanisms have been proposed, for example, electrostatic shock acceleration (ESA) [5-9], target normal sheath acceleration [10-13], magnetic vortex acceleration [14-18], and radiation pressure acceleration (RPA) [19-41], etc. Among these mechanisms, the RPA is regarded as one of the most promising schemes because of its high energy conversion efficiency and the excellent quality of the accelerated ions $[19,29,30]$. When the radiation pressure is dominant, the electrons pile up by

\footnotetext{
*Corresponding author. hxr_nwnu@163.com

Published by the American Physical Society under the terms of the Creative Commons Attribution 4.0 International license. Further distribution of this work must maintain attribution to the author(s) and the published article's title, journal citation, and DOI.
}

the radiation pressure and form the compressed electron layer. The laser-driven ion acceleration arises from the charge separation field caused by the ultraintense laser pushing the compressed electron layer forward [19]. The ions under this strong charge separation field could be rapidly accelerated and catch up with the fast-moving compressed electron layer. The laser pulse would penetrate into the target, which is regarded as the hole-boring (HB) stage $[31,37]$. Until the laser penetrates through the target, the RPA structure that the compressed electron layer overlaps the accelerated ion layer as a whole and forms a quasielectric-neutral structure, which is also called plasma mirror, detaches from the target and is accelerated to high energy by continuously reflecting laser, and this process is called the light-sail stage [35,37].

However, there are also some problems in the RPA regime, such as the maximum energy limitation of the accelerated ions [39] and the transverse instability. There have been many researches about the transverse instability $[22,24-26]$, and a recent work indicates that the ripples on the target is induced by the coupling between oscillating electrons and quasistatic ions [42]. On the other hand, in the high dimension situation, the amplitude profile of the normal transversely Gaussian laser has a transverse distribution, which means a transversely nonuniform radiation on the foil. Therefore, the common flat foil target is doomed to curve by the transversely nonuniform acceleration. The curved foil would contribute to a rapid foil 
deformation and premature determination of the ion acceleration $[22,24]$.

In order to overcome the target curve and improve the quality of the accelerated ion beam, a series of schemes have been proposed so far. Some schemes aimed at shaping the laser pulse to get a steep-front laser [38], or using the specific combination of different laser modes [24]. Other schemes tended to construct the specific target structure, such as the shaped foil target [28], the double layer target [36], and the mixed multispecies foil target [34]. However, in these previous schemes, the advanced target fabrication technology and rigorous laser shaping technology are indispensable. Therefore, it is a challenge to realize these schemes in ordinary laboratory conditions. If we can realize a high quality ion acceleration by using the normal transversely Gaussian laser and the common flat foil target, which could be respectively easier obtained in an ordinary laboratory, the requirement of experiment condition could be vastly reduced.

For the above consideration, we propose a new scheme to generate high quality ion beams by using the normal transversely Gaussian laser and the common flat foil target. Since the curve of the foil target is attributed to the nonuniform radiation of the single transversely Gaussian laser pulse on the foil target, we could add another counterpropagating transversely Gaussian laser pulse from the other side of the foil to counteract the nonuniform radiation on the foil, which may effectively suppress the target curve and improve the quality of the accelerated ions.

The organization of this paper is as follows. In Sec. II, we propose our scheme and come up with the corresponding optimum parameters condition for the auxiliary laser. In Sec. III, the 2D particle-in-cell (PIC) simulations are performed to verify our scheme by using the relativistic electromagnetic PIC code VORPAL [43]. In addition, the effects of different parameters and the arrival time of the auxiliary laser are also investigated. At last, the conclusion is presented in Sec. IV.

\section{MODEL AND THEORETICAL ANALYSIS}

In order to counteract the nonuniform radiation on a common flat foil while being radiated by a single transversely Gaussian laser, we add another counterpropagating transversely Gaussian laser pulse from the other side of the foil, as shown in Fig. 1. In the schematic diagram, the left and right lasers are the main and auxiliary pulses, respectively. If the parameters of the auxiliary lasers satisfy an appropriate condition, the longitudinal component of the resultant ponderomotive force of these two lasers would vary so slowly in the transverse direction, and the foil could be relatively uniformly accelerated, which can effectively suppress the curve of the foil. A high quality ion beam can be generated through an efficient RPA of the foil. In consequence, after given the main laser, the parameters of

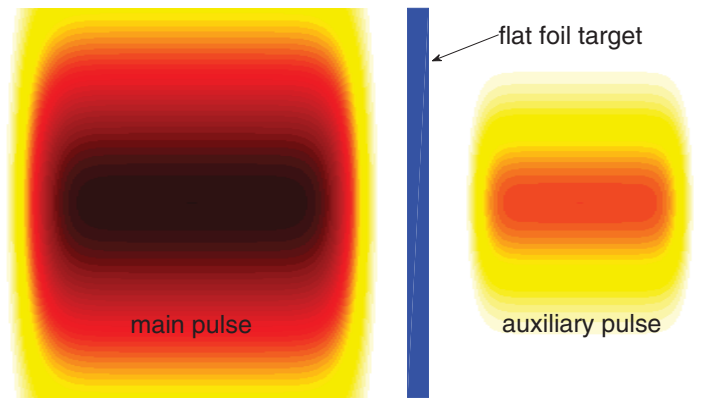

FIG. 1. Schematic diagram of our scheme that two matched counterpropagating transversely Gaussian lasers impinge on a flat foil target.

the matched auxiliary laser are what we should investigate in this part.

When a laser impinges on a target, the relativistic ponderomotive force on the electron can be given by $[4,44]$

$$
\mathbf{F}=-m_{e} c^{2} \nabla \gamma
$$

where $m_{e}$ and $c$ are the rest electron mass and vacuum light speed, $\gamma=\sqrt{1+a^{2}}$ is the relativistic factor, and $a=e E / m_{e} \omega c$ represents the normalized laser amplitude, where $-e$ is the electron charge, $E$ and $\omega$ are the electric field amplitude and frequency of the laser, respectively. In the ultralaser intensity region, i.e., $\sqrt{1+a^{2}} \approx a$ while $a \gg 1$, the longitudinal component $(x)$ of the ponderomotive force is

$$
F_{x}=-\frac{m_{e} c^{2}}{2 a} \frac{\partial a^{2}}{\partial x}
$$

In consequence, longitudinal component $(x)$ of the resultant ponderomotive force of these two lasers is

$$
\begin{aligned}
f_{x}= & F_{m x}+F_{a x}=-\frac{m_{e} c^{2}}{2 a_{m}} \frac{\partial a_{m}{ }^{2}}{\partial x} \\
& -\frac{m_{e} c^{2}}{2 a_{a}} \frac{\partial a_{a}{ }^{2}}{\partial x},
\end{aligned}
$$

where the subscripts $m$ and $a$ represent the main and auxiliary lasers, respectively.

It is assumed that the main and auxiliary lasers are both transversely Gaussian, and emit from the front and rear surfaces of the foil target, respectively. The expressions of the main and auxiliary lasers are

$$
\begin{aligned}
a_{m}= & a_{1} \exp \left[-(1 / 2)\left(y / r_{1}\right)^{2}\right] \\
& \times \exp \left\{-(1 / 2)\left[\left(x-c t-\left(x_{0}-l_{0} / 2\right)+\delta_{1}\right) / L_{1}\right]^{10}\right\},
\end{aligned}
$$

and 


$$
\begin{aligned}
a_{a}= & a_{2} \exp \left[-(1 / 2)\left(y / r_{2}\right)^{2}\right] \\
& \times \exp \left\{-(1 / 2)\left[\left(x+c t-\left(x_{0}+l_{0} / 2\right)-\delta_{2}\right) / L_{2}\right]^{10}\right\},
\end{aligned}
$$

respectively, where $a_{1}$ and $a_{2}$ are the normalized amplitudes, $r_{1}$ and $r_{2}$ are the waist radiuses, $L_{1}=c t_{1}$ and $L_{2}=$ $c t_{2}$ are the pulse widths, $\delta_{1}$ and $\delta_{2}$ are the longitudinal laser envelope factors, respectively. The center of the foil target is in $x=x_{0}$ and the target thickness is $l_{0}$.

At $t=0$, the main and auxiliary lasers impinge on the foil at the meantime. They start respective HB stages at the front and rear sides of the foil. In each HB process, the initially rest ions in the compressed electron layer would be accelerated to twice $\mathrm{HB}$ velocity, which is known as the electrostatic shock acceleration (ESA) [21,23]. In consequence, there emerges two countermoving ion beams in the target, one is accelerated forward and the other is accelerated backward. The moment when these two countermoving ion beams meet in the target interior is approximately regarded as the moment that the coaction effect of these two lasers starts to work, which can be written as

$$
t_{c}=\frac{l_{0}}{2\left(v_{b 1}+v_{b 2}\right)},
$$

where $v_{b 1}$ and $v_{b 2}$ are the $\mathrm{HB}$ velocities of the main and auxiliary lasers, respectively. It is known that the relativistic HB velocity $v_{b}$ is [32]

$$
\frac{v_{b}}{c}=\frac{\Xi^{1 / 2}}{1+\Xi^{1 / 2}},
$$

with $\Xi=\frac{Z m_{e} n_{c}}{A m_{p} n_{e}} a^{2}$, where $Z$ and $A$ are the nuclear charge number and relative atomic mass of the ion, $m_{p}$ is the mass of the proton, $n_{e}$ is the electron density of the foil target, and $n_{c}=m_{e} \omega^{2} / 4 \pi e^{2}$ is the critical density, respectively.

On the other hand, at $t=t_{c}$, the moment when the coaction effect of these two lasers starts to work, the positions of the main and auxiliary laser fronts are $x_{1}=$ $x_{0}-l_{0} / 2+v_{b 1} t_{c}$ and $x_{2}=x_{0}+l_{0} / 2-v_{b 2} t_{c}$, respectively.

By setting $x=x_{1}, t=t_{c}$ in Eq. (4), $x=x_{2}, t=t_{c}$ in Eq. (5), substituting Eqs. (4) and (5) into Eq. (3), and letting $a_{2}=\alpha a_{1}, r_{2}=\xi r_{1}, L_{2}=\eta L_{1}$, one can obtain the longitudinal component $(x)$ of resultant ponderomotive force on the electron layer at the moment when the coaction effect of these two lasers starts to work as

$$
\begin{aligned}
f_{x}= & 5 m_{e} c^{2}\left\{\frac{a_{1}}{L_{1}} s_{1} \exp \left[-(1 / 2)\left(y / r_{1}\right)^{2}\right]\right. \\
& \left.-\frac{\alpha a_{1}}{\eta L_{1}} s_{2} \exp \left[-(1 / 2)\left(y / \xi r_{1}\right)^{2}\right]\right\},
\end{aligned}
$$

where $s_{1}=\exp \left\{-(1 / 2)\left\{\left[\left(c-v_{b 1}\right) t_{c}+\delta_{1}\right] / L_{1}\right\}{ }^{10}\right\}\left\{\left[\left(c-v_{b 1}\right)\right.\right.$ $\left.\left.t_{c}+\delta_{1}\right] / L_{1}\right\}^{9}, s_{2}=\exp \left\{-(1 / 2)\left\{\left[\left(c-v_{b 2}\right) t_{c}-\delta_{2}\right] / \eta L_{1}\right\}^{10}\right\}$ $\left\{\left[\left(c-v_{b 2}\right) t_{c}-\delta_{2}\right] / \eta L_{1}\right\}^{9}$, and $\alpha, \xi, \eta$ are the scale factors, respectively. After taking the derivative of Eq. (8) with respect to $y$, one can get the transverse variation of the $f_{p_{x}}$ as

$$
\begin{aligned}
\frac{\partial f_{x}}{\partial y}= & K s_{1} y \exp \left[-1 / 2\left(y / r_{1}\right)^{2}\right] \\
& \times\left\{1-\frac{\alpha s_{2}(\eta)}{\xi^{2} \eta s_{1}} \exp \left[-1 / 2\left(y / r^{\prime}\right)^{2}\right]\right\},
\end{aligned}
$$

where $K=5 m_{e} c^{2}\left[a_{1} /\left(r_{1}{ }^{2} L_{1}\right)\right]$, and $r^{2}=\left[\xi^{2} /\left(1-\xi^{2}\right)\right] r_{1}{ }^{2}$. For the purpose of suppressing the curve and realizing an efficient RPA of the foil, the intensity and the waist radius of the auxiliary laser should be less than those of the main laser, i.e., $0<\alpha<1$ and $0<\xi<1$. In the meanwhile, in order to avoid additional disturbance of the auxiliary laser to the acceleration of the foil, the pulse width of the auxiliary laser should not be too long, which leads to $0<\eta<1$.

If the longitudinal component of the resultant ponderomotive force varies so slowly in the transverse direction, i.e., $\frac{\partial f_{x}}{\partial y} \sim 0$ in Eq. (9), the curve of the foil can be effectively suppressed. Therefore, the matching condition of these two lasers can be obtained as

$$
\alpha s_{2}(\eta) /\left(\xi^{2} \eta s_{1}\right) \sim 1
$$

According to the matching condition of Eq. (10), by giving the parameters of the main laser, one can get the corresponding parameters of the matched auxiliary laser. For example, by setting $a_{1}=120, \quad r_{1}=10 \lambda, \quad \delta_{1}=$ $L_{1}=8 \lambda, a_{2}=30$, and $\delta_{2}=L_{2}=5 \lambda, l_{0}=0.4 \lambda, n_{e}=$ $100 n_{c}$, where $\lambda$ is the laser wavelength, one can get the optimum waist radius of the auxiliary laser $r_{2}=\eta r_{1}=5 \lambda$ from the matching condition, which is also verified by the following PIC simulation in Sec. III.

However, if the parameters of the auxiliary laser can not satisfy the theoretical optimums, the coaction effect of these two lasers is what we also should investigate here. Given the parameters of the main pulse, the transverse distributions of the longitudinal component of the resultant ponderomotive force with different intensities $a_{2}$, waist radiuses $r_{2}$, and pulse widths $L_{2}$ of the auxiliary laser are presented in Fig. 2. In the single laser situation, the transverse distribution of the longitudinal component of the resultant ponderomotive force is apparently exponential, which leads to a transversely nonuniform acceleration of the foil. However, by adding another auxiliary laser from the other side of the foil, the transversely nonuniform distribution of the longitudinal component of the resultant ponderomotive force would be apparently counteracted. In the above discussion, for $a_{1}=120, r_{1}=10 \lambda, \delta_{1}=$ $L_{1}=8 \lambda, l_{0}=0.4 \lambda$, and $n_{e}=100 n_{c}$, the theoretical optimum 

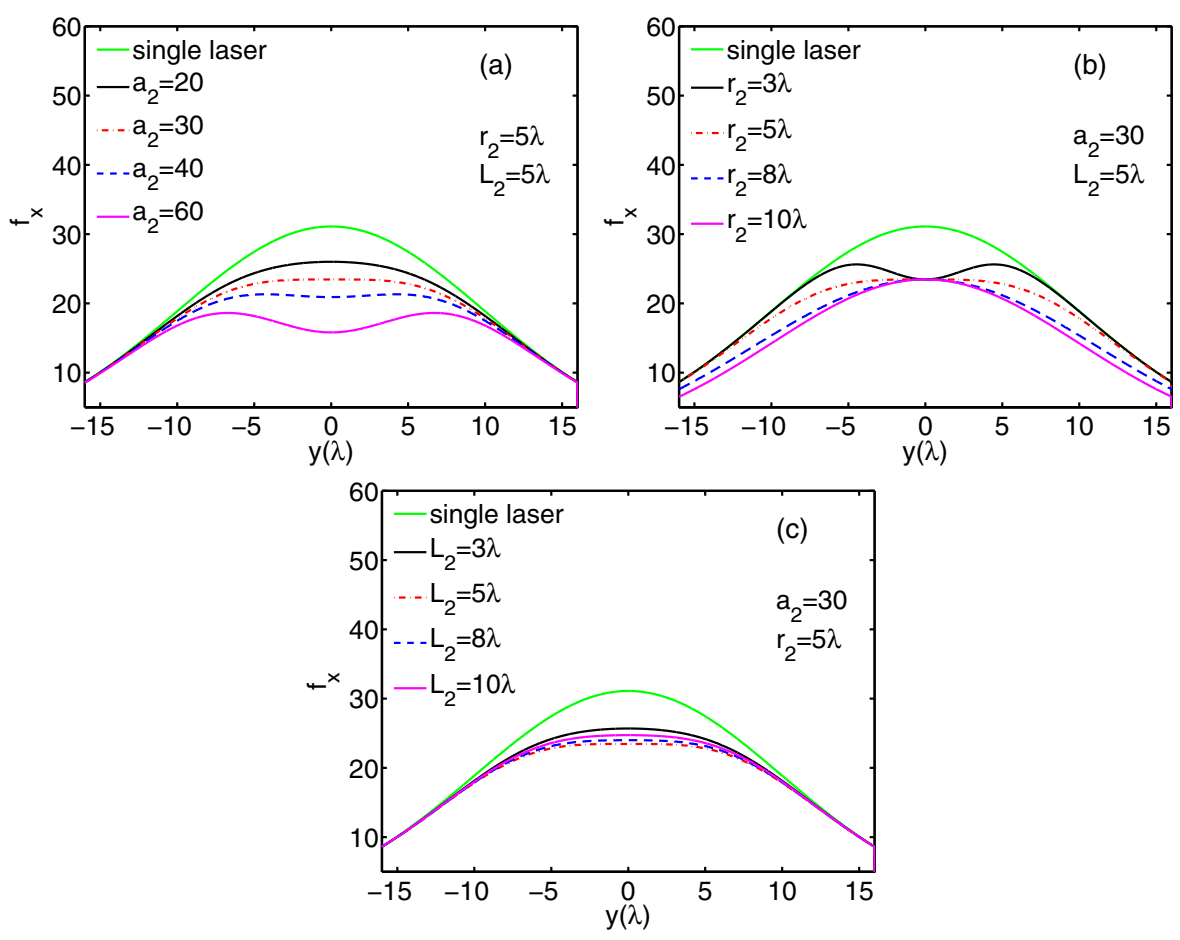

FIG. 2. The transverse distributions of the longitudinal component ( $x$ ) of the resultant ponderomotive force for different intensities (a), waist radiuses (b), and pulse widths (c) of the auxiliary laser. For the main laser pulse, normalized amplitude $a_{1}=120$, waist radiuses $r_{1}=10 \lambda$, and pulse width $L_{1}=8 \lambda$.

parameters of the auxiliary laser are $a_{2}=30, r_{2}=5 \lambda$, and $\delta_{2}=L_{2}=5 \lambda$. It can be found that in Figs. 2(a) and 2(b), this set of optimum parameters is also the critical boundary of two suppression situations. The first one that the transverse distribution of the longitudinal component of resultant ponderomotive force shows a concave-up function, is called oversuppression situation, in which the resultant acceleration on the center area is lower than that on the vicinity area. Therefore, a reverse cone-shape RPA structure would be formed in this situation. The second one that the transverse distribution of the longitudinal component of resultant ponderomotive force shows a concave-down function, is called undersuppression situation, in which the transversely Gaussian-like distribution of the longitudinal component of resultant ponderomotive force on the foil still exists. No matter in the oversuppression or undersuppression situations, the curve of the foil still would develop in the acceleration process. However, by varying the pulse width of the auxiliary laser, the differences are nearly negligible, as seen in Fig. 2(c). In fact, the pulse width of the auxiliary laser, which determines how long the suppression effect would keep on, would have some influence in our scheme, which is also investigated by PIC simulations and detailed analyses in Sec. III.

It should be noticed that the above theoretical analysis is based on the condition that we do not take into account the arrival time deviation of these two lasers, which means two lasers impinge on the flat foil target simultaneously.
In some way, the arrival time deviation would have an influence on the quality of the accelerated ions, the corresponding PIC simulation and detailed analysis are also presented at the end of Sec. III.

\section{SIMULATION AND RESULTS}

Basing on the above theoretical analysis, the 2D PIC simulations are performed to verify our scheme and investigate the effects of different parameters of the auxiliary laser on the acceleration. The length of the simulation box is $111 \mu \mathrm{m} \times 32 \mu \mathrm{m}$, and the grid resolution is $0.01 \mu \mathrm{m}$. The main and auxiliary lasers are all transversely circularly polarized and have the same wavelength $\lambda=1 \mu \mathrm{m}$, which corresponds to the laser period $T_{L}=3.3 \mathrm{fs}$. For the two lasers case, the optimum parameters presented in Sec. II are used here first, i.e., $a_{1}=120, a_{2}=30, L_{1}=\delta_{1}=8 \lambda$, $L_{2}=\delta_{2}=5 \lambda, r_{1}=10 \lambda$, and $r_{2}=5 \lambda$. The ultrathin foil target, which consists of protons and electrons with $n_{e}=100 n_{c}$, is located in $22.3 \mu \mathrm{m}<x<22.7 \mu \mathrm{m}$, i.e., target thickness $l_{0}=0.4 \mu \mathrm{m}$. In all simulations, the normalized amplitude of the main laser pulse, the target thickness and initial electron density of the target satisfy the optimum target thickness relation [29] as

$$
\frac{l_{0}}{\lambda}=\frac{1}{2 \pi} \frac{n_{c}}{n_{e}} a_{1} .
$$

At $t=0$, the main and auxiliary lasers reach the foil target. On the other hand, a comparison simulation is also performed 

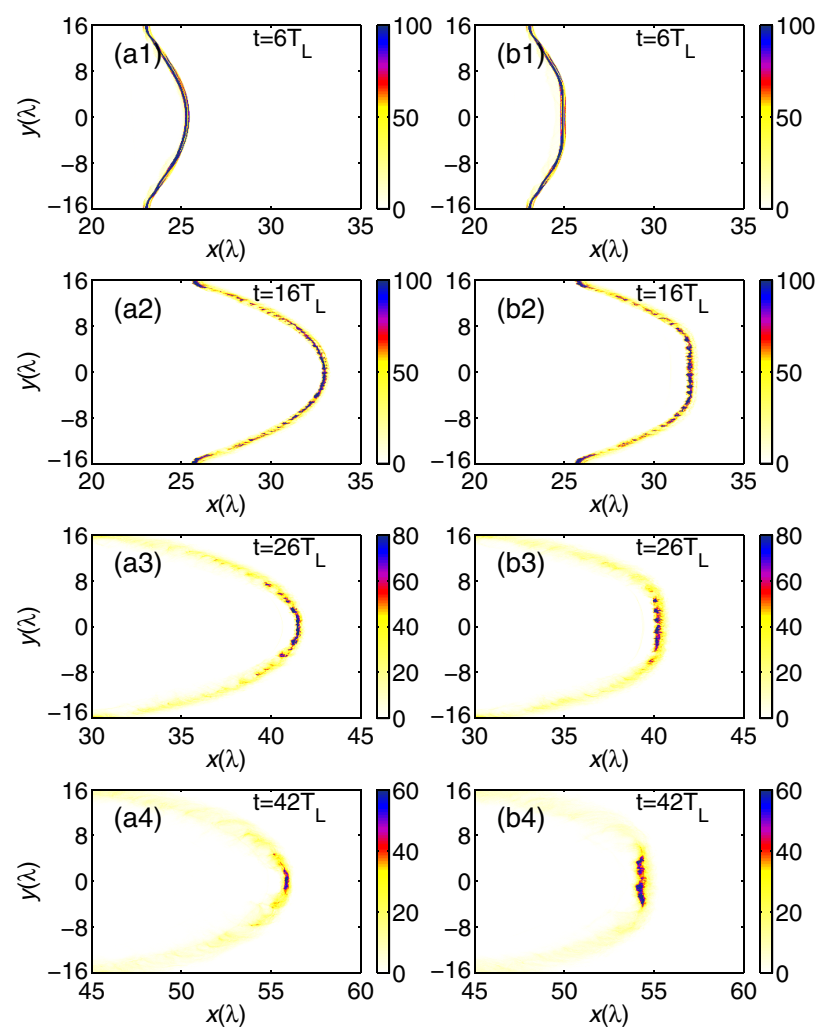

FIG. 3. The spatial density distributions (normalized by $n_{c}$ ) of the proton for the single laser case (a1)-(a4) and two lasers case (b1)-(b4). These snapshots are corresponding to four specific moments, $t=6 T_{L}, t=16 T_{L}, t=26 T_{L}$, and $t=42 T_{L}$.

here, i.e., there is no auxiliary laser in this comparison simulation, and other simulation parameters are the same with the two lasers case.

The evolution of the spatial proton density distributions for the single laser case and two lasers case are presented in Fig. 3. The left column shows the snapshots of the single laser case in four specific moments, and the right column is of the two lasers case. For the single laser case, the target starts to curve at the beginning of the acceleration, due to the transversely nonuniform radiation by the main pulse, as expected in Eq. (2). As the process of the acceleration, the curve becomes worse, and the transverse instability gradually emerges on the target in Fig. 3 (a2). Then, the curve target allows the proton diffusion from the center to the wings, as shown in Fig. 3 (a3). Even if the proton diffusion could relieve the transverse instability, it inevitably lead to the fracture of the target in the meantime. The diffusion of protons would continue, until the target becomes transparent and the acceleration is terminated. However, for the two lasers case, it can be found that the target curve is suppressed effectively at the beginning of the acceleration in Fig. 3 (b1). The central part of the target is accelerated uniformly by the collaboration of these two matched lasers. Even the transverse instability still emerges on the foil, the flat acceleration structure could also availably restrain the
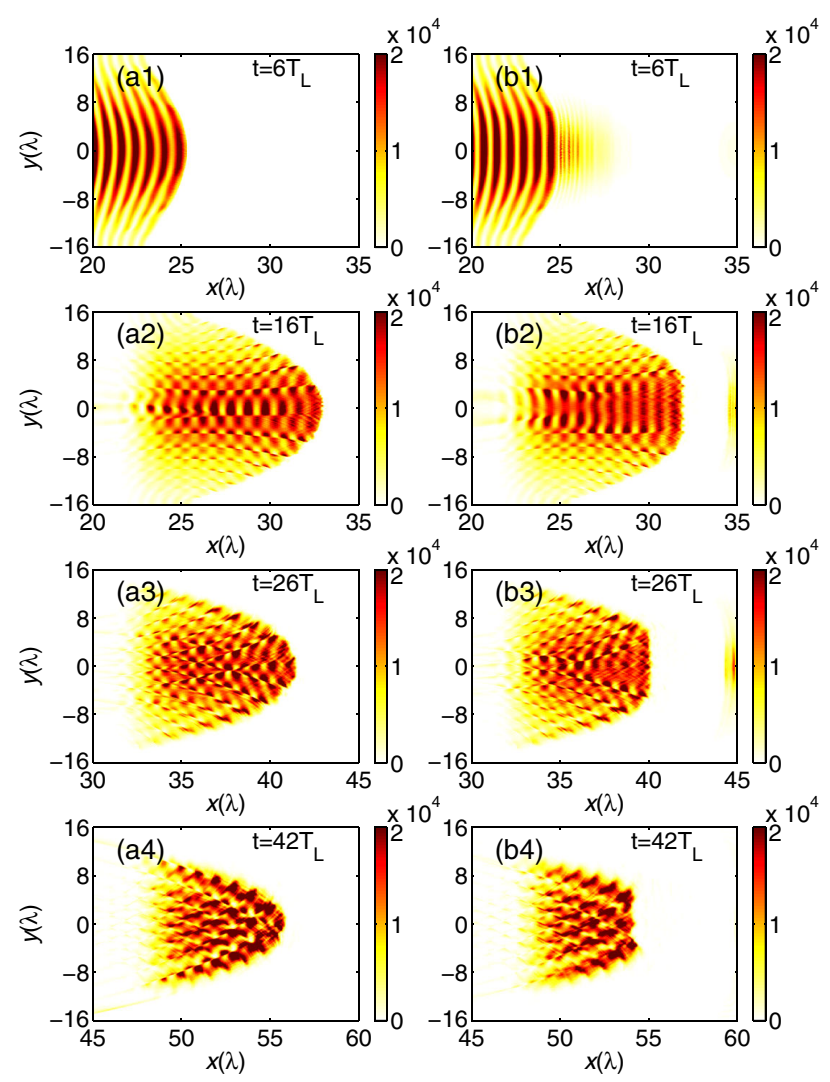

FIG. 4. The dimensionless spatial laser intensity distributions for the single laser case (a1)-(a4) and two lasers case (b1)-(b4). These snapshots are corresponding to four specific moments, $t=6 T_{L}, t=16 T_{L}, t=26 T_{L}$, and $t=42 T_{L}$.

proton diffusion in the central part, as presented in Fig. 3 (b2) and (b3). Therefore, the RPA structure could be held together and accelerated uniformly to high energy.

Similarly, the evolution of the dimensionless spatial laser intensity distributions for the single laser case and two lasers case are presented in Fig. 4. The left column shows the snapshots of the single laser case in four specific moments, and the right column is of the two lasers case. In the single laser case, the target is inevitably curved under the transversely nonuniform radiation. This curve structure, just like a concave plasma mirror, could focus the laser toward the axis, and there emerges a ripplelike structure in the intensity distribution. Hence, such an intensity distribution would reinforce the transverse nonuniform radiation on the foil and boost the proton diffusion, as seen in Fig. 4 (a1)-(a4). However, in the two lasers case, the curve of the foil can be effectively suppressed at the beginning of the acceleration, and the target can be relatively uniformly accelerated by these two lasers. On the other hand, such a flat acceleration structure could also modulate the main pulse in return. It can be seen that in Fig. 4 (b2)-(b3), the central part of the main pulse has been modulated into a flattop profile. Therefore, the longitudinal ponderomotive force on the foil will vary slowly in the transverse direction, 

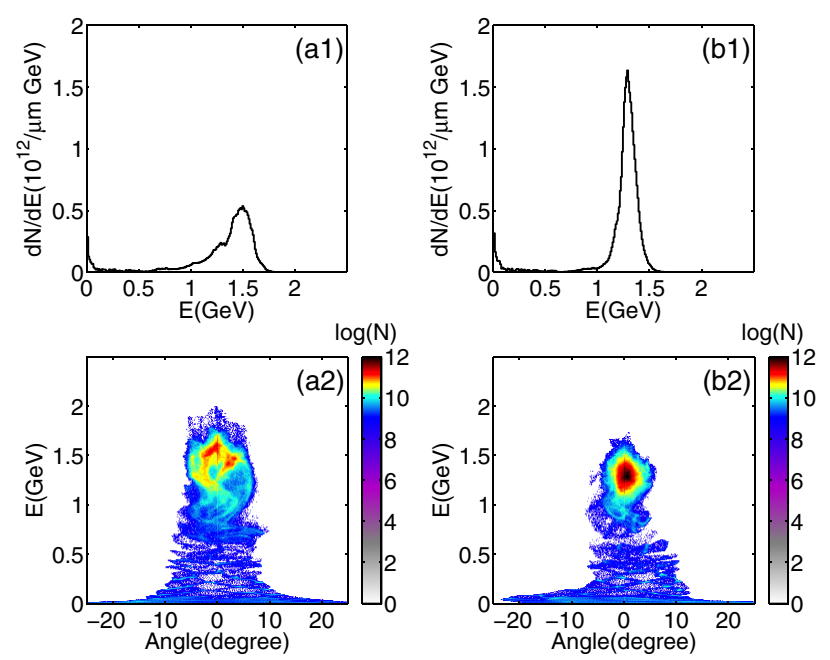

FIG. 5. The energy spectra of the proton in the single laser case (a1) and two lasers case (b1). The energy angular distributions of the proton in the single laser case (a2) and two lasers case (b2). Here, we choose those protons who locate in the region $-4 \mu \mathrm{m} \leq$ $y \leq 4 \mu \mathrm{m}$ as the objects of statistics. These snapshots are corresponding to the moment $t=42 T_{L}$.

and the foil can still be accelerated uniformly by the main pulse after the auxiliary laser passing through.

The energy spectra and the energy angular distributions of the protons at $t=42 T_{L}$ for the single laser and two lasers cases are presented in Fig. 5. The left column is the results of the single laser case, and the right column is of the two lasers case. For the single laser case, due to the curved accelerated RPA structure, the electrons would continually escape from the center to the wings and take away a part of the fast protons. Therefore, the quantity of accelerated protons in the center area is inevitable decreased, as shown in Fig. 5 (a1). In the meanwhile, the curved foil forms a natural cone structure, which guides the motion of the protons and enlarges the angular divergency of the fast protons, about from $-7^{\circ}$ to $7^{\circ}$, as shown in Fig. 5 (a2). However, for the two lasers case, due to the effective suppression of the target curve and the uniform high efficient RPA of the foil, a high quality monoenergetic proton beam is generated finally. It can be seen that in the Fig. 5 (b1), the energy peak of the accelerated protons is about $1.3 \mathrm{GeV}$ with the full-width-at-half-maximum (FWHM) range from $1.2 \mathrm{GeV} \sim 1.4 \mathrm{GeV}$. The number of the accelerated protons in the statistical region, $-4 \mu \mathrm{m} \leq$ $y \leq 4 \mu \mathrm{m}$, is around $3.5 \times 10^{12} / \mu \mathrm{m}$, and the energy conversion efficiency from laser to these protons is about $20.8 \%$. Furthermore, the accelerated proton beam is of a high collimation with the angular divergency less than $3^{\circ}$, as shown in Fig. 5 (b2).

Until now, by the 2D PIC simulation, we have proved that high quality ion beams can be generated through a relatively more uniform RPA of the flat foil target by adding another matched transversely Gaussian auxiliary laser.
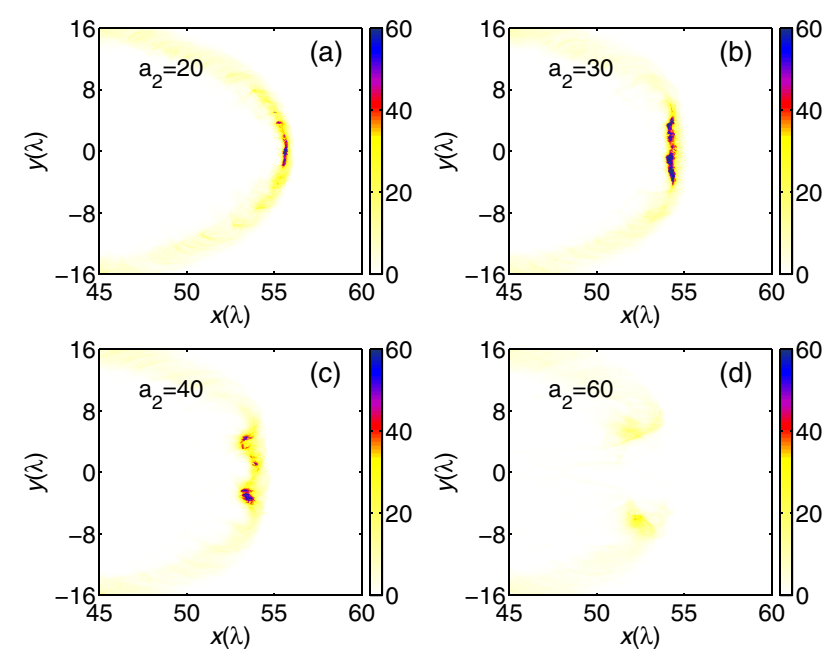

FIG. 6. The spatial density (normalized by $n_{c}$ ) distributions of the proton with different intensities of the auxiliary laser. The normalized amplitude of the auxiliary laser $a_{2}=20$ in (a), $a_{2}=$ 30 in (b), $a_{2}=40$ in (c), and $a_{2}=60$ in (d). These snapshots are corresponding to the moment $t=42 T_{L}$.

In the following part, we would investigate how these parameters of the auxiliary laser, e.g., the intensity, waist radius and pulse width, affect the quality of accelerated protons in our scheme.

For the intensity effects of the auxiliary laser, four comparative simulations are performed by varying the intensity of the auxiliary laser as $a_{2}=20,30,40$, and 60 labeled with case (a), (b), (c), and (d), respectively. The other parameters are the same with the optimum parameters. The spatial proton density distributions of these four cases at $t=42 T_{L}$ are presented in Fig. 6. For case (a), the longitudinal component of the resultant ponderomotive force represents a transversely nonuniform distribution, as shown in Fig. 2(a), the foil is inevitably curved due to the undersuppression of the auxiliary laser, as seen in Fig. 6(a). For case (b), by using the optimum parameters, it can be found that the foil is uniformly accelerated and there emerges a flat RPA structure in Fig. 6(b). For case (c), it can be found that in Fig. 6(c), the spatial proton density distribution presented a reverse cone shape because of the oversuppression of the auxiliary laser. For case (d), the transverse distributions of the longitudinal component of the resultant ponderomotive force on the center area presented a reverse cone shape, as shown in Fig. 2(a), which means the longitudinal acceleration on the center area $-5 \lambda<y<5 \lambda$ is lower than the vicinity area. In consequence, the foil is "reverse-drilled" in the acceleration process, as shown in Fig. 6(d).

For the waist radius effects of the auxiliary laser, four comparative simulations have been done by varying the waist radius of the auxiliary laser as $r_{2}=3 \lambda, 5 \lambda, 8 \lambda$, and $10 \lambda$ labeled with case (a), (b), (c), and (d), respectively. The other parameters are the same with the optimum parameters. The spatial proton density distributions of 

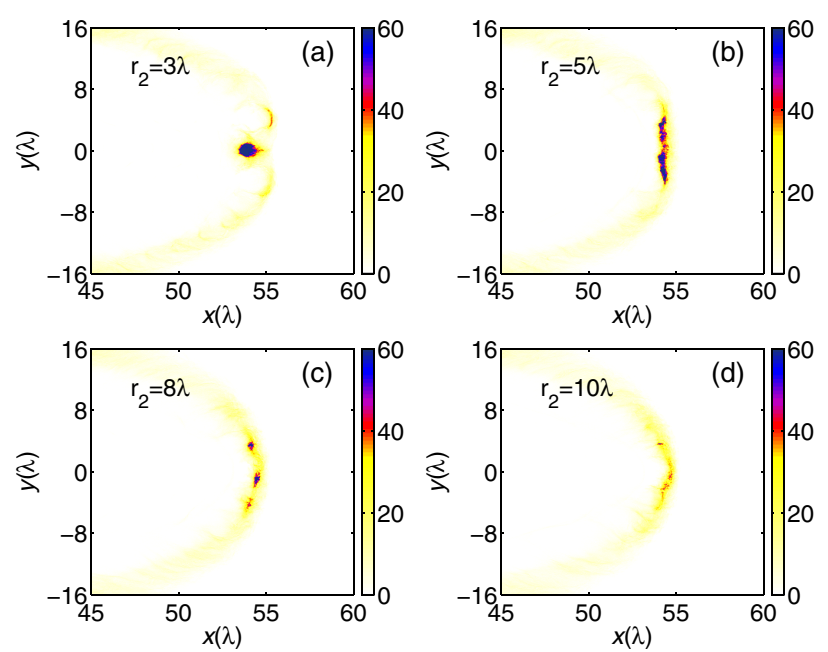

FIG. 7. The spatial density (normalized by $n_{c}$ ) distributions of the proton with different waist radiuses of the auxiliary laser. The waist radius $r_{2}=3 \lambda$ in (a), $r_{2}=5 \lambda$ in (b), $r_{2}=8 \lambda$ in (c), and $r_{2}=10 \lambda$ in $(\mathrm{d})$, respectively. These snapshots are corresponding to the moment $t=42 T_{L}$.

these four cases at $t=42 T_{L}$ are presented in Fig. 7. For case (a), as expected in Fig. 2(b), due to the oversuppression on the foil, the acceleration of the center of the foil is less than that of the vicinity. Therefore, the deformation of the foil forms a reverse cone-shape acceleration structure, which could trap the fast protons in the center region and prevent them from escaping, as shown in Fig. 7(a). But, after the electron layer in the vicinity become transparent, the laser could penetrate through the RPA structure from the transparent region, which would lead to the leak of the light sail and prematurely terminate the acceleration. For case (b), $r_{2}=5 \lambda$ is the optimum waist radius from the theoretical analysis results. The nonuniform acceleration distribution on the foil is effectively counteracted by the auxiliary laser. In consequence, the foil is uniform accelerated and a nearly flat RPA structure can be seen in Fig. 7(b). For case (c), also as expected in Fig. 2(b), due to the undersuppression on the foil, the auxiliary laser did not completely counteract the transversely nonuniform radiation of the main laser on the foil. As a result, the foil is inevitably curved due to the nonuniform acceleration, as shown in Fig. 7(c). Alike, for case (d), due to the more inefficient counteracting effect as presented in Fig. 2(b), the curve of the foil is worse than case (c), as shown in Fig. 7(d).

For the pulse width effects of the auxiliary laser, four comparative simulations are also performed by varying the pulse width of the auxiliary laser as $L_{2}=3 \lambda, 5 \lambda, 8 \lambda$, and $10 \lambda$ labeled with case (a), (b), (c), and (d), respectively. The other parameters are the same with the optimum parameters. The spatial proton density distributions of these four cases at $t=42 T_{L}$ are presented in Fig. 8. For case (a) and case (b), in which the pulse width $L_{2}<L_{1}$, under an appropriate duration of the suppression effect, the flat RPA
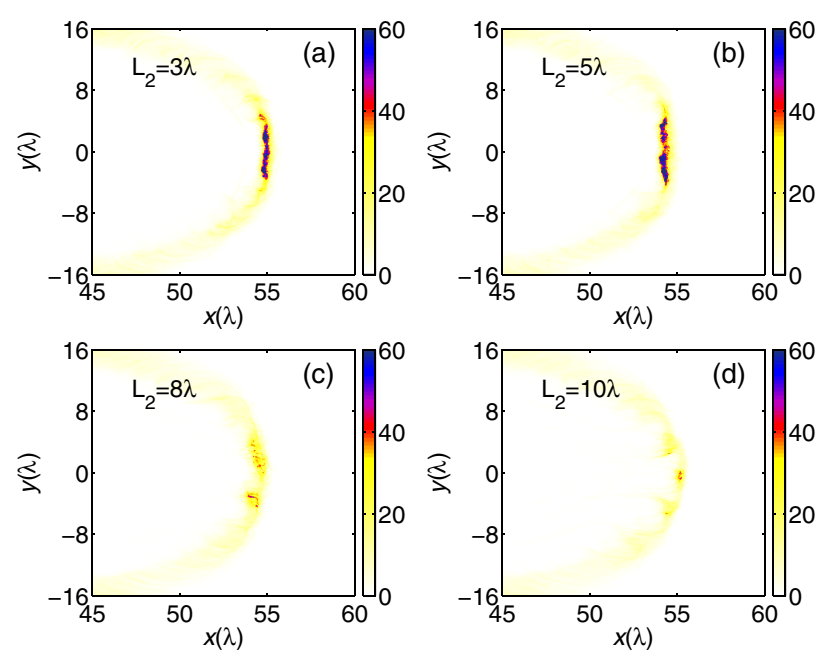

FIG. 8. The spatial density (normalized by $n_{c}$ ) distributions of the proton with different pulse widths of the auxiliary laser. The pulse width of the auxiliary laser $L_{2}=3 \lambda$ in (a), $L_{2}=5 \lambda$ in (b), $L_{2}=8 \lambda$ in (c), and $L_{2}=10 \lambda$ in (d), respectively. These snapshots are corresponding to the moment $t=42 T_{L}$.

structure is still concentrated together, as seen in Figs. 8(a) and 8 (b). However, due to the relatively shorter suppression duration, the flatness of the RPA structure in case (a) is a little bit less than that in case (b). But for case (c) and case (d), in which the pulse width $L_{2} \geq L_{1}$, the accelerated protons are dispersed and the main RPA structure becomes indistinct in Figs. 8(c) and 8(d). It can be found that, by comparing Figs. 8(c) and 8(d), the longer the pulse width of the auxiliary laser is, the worse the RPA structure becomes. Therefore, the pulse width of the auxiliary laser would affect the acceleration of the protons. If the pulse width is too short, the counteracting effect cannot last long enough, which leads to an incomplete suppression on the curve of the foil. On the other hand, if the pulse width is too long, the auxiliary laser can do damage to the RPA structure by the additional disturbance to the RPA structure. As we know, the accelerated protons are continually oscillating in the RPA field [33], the additional disturbance of the auxiliary laser may push them out of the RPA potential well and make them fall behind. According to the simulation results, the optimum pulse width of the auxiliary laser is $\eta \sim 5 / 8$.

Up to now, we assumed that the main and auxiliary lasers reach the foil target at the meantime. If there is an arrival time deviation of these two lasers, there should be an influence on the quality of the accelerated ion beam.

For the time deviation effects, four comparative simulations are also done by varying the arrival time of the auxiliary laser and the other parameters are still the same as those of the optimum parameters. We have set that the main laser reaches the foil at $t=0$. The auxiliary laser reaches the foil at $t=-4 T_{L}, t=-T_{L}, t=0$, and $t=4 T_{L}$ in these four cases, respectively. The number density angular distributions of the accelerated protons are presented in 

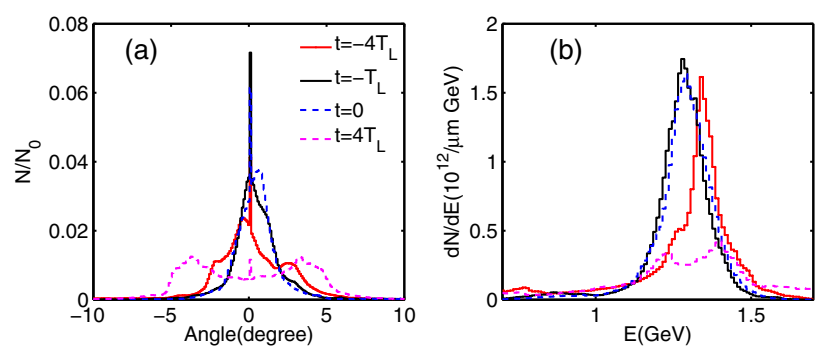

FIG. 9. The number density (normalized by the amount of statistical protons) angular distributions (a) and the energy spectra (b) of the proton with different arrival times of the auxiliary laser. In the four comparative simulations, the auxiliary laser pulse reaches the target at $t=-4 T_{L}, t=-T_{L}, t=0$, and $t=4 T_{L}$, respectively. Here, we choose those protons who locate in the region $-4 \mu \mathrm{m} \leq y \leq 4 \mu \mathrm{m}$ as the objects of statistics. These snapshots are corresponding to the moment $t=42 T_{L}$.

Fig. 9(a). For the $t=-T_{L}$ and $t=0$ cases, the main and auxiliary lasers reach the foil almost simultaneously. The counteract effect would work effectively and the predeformation of the foil can be effectively avoided. Therefore, the nonuniform acceleration of the foil can be efficiently counteracted, and the accelerated ion beam with a highly collimation is obtained finally, as shown in Fig. 9(a). For the $t=-4 T_{L}$ and $t=4 T_{L}$ cases, there is obvious time deviation between the arrival times of the main and auxiliary lasers, which lead to a predeformation on the foil by the relatively earlier arrived pulse. The predeformation is adverse to the RPA process and would worsen the collimation of the accelerated ion beam, as seen in Fig. 9(a).

The energy spectra of the accelerated protons with different arrival times of the auxiliary laser are presented in Fig. 9(b). For the $t=-4 T_{L}$ case, the auxiliary laser reaches the foil much earlier than the main laser. The transversely Gaussian auxiliary laser would also cause a reverse cone-shape curve on the foil. A part of the protons would be backward accelerated before the main pulse reach the foil and stay in the low energy region. In consequence, the quantity of the efficiently accelerated ion is declined, which leads to a slightly energy up-shift of the energy peak, as shown in Fig. 9(b). For the $t=-T_{L}$ case, the auxiliary laser reaches the foil a little bit earlier than the main laser, and starts the hole-boring process on the foil. Therefore, the counteracting effect would work as soon as the main laser reaches the target, and the curve of the foil can be effectively suppressed in time. As a result, a high quality and collimation proton beam can be generated finally. For the $t=0$ case, the main and auxiliary lasers reach the foil at the meantime. But, due to the definite foil thickness, there would be a hole-boring stage before the counteracting effect of the auxiliary laser starts to work. As a result, the foil would be slightly curved and the quality of the accelerated protons becomes a little bit worse than that of the $t=-T_{L}$ case, in which the counteracting effect of the auxiliary laser starts to work just before the predeformation by the main laser. For the $t=4 T_{L}$ case, the auxiliary laser reaches the foil after the deformation of the foil. The curve of the foil cannot be eliminated after the deformation. The fast protons will continually escape from the wings. Therefore, the quality of the accelerated proton beam is inevitably dropped, as seen in Fig. 9(b).

\section{CONCLUSION}

In conclusion, for the purpose of generating high quality ion beams by overcoming the transversely nonuniform RPA of a common flat foil while been radiated by a single transversely Gaussian laser, we proposed to add another matched counterpropagating transversely Gaussian auxiliary laser to counteract the transversely nonuniform radiation on the foil. Considering the longitudinal component of the resultant ponderomotive force of these two lasers should vary slowly in the transverse direction, the matching condition of these two lasers is obtained. According to the matching condition, the optimum parameters of the auxiliary laser could be obtained after given the parameters of the main pulse. The corresponding 2D PIC simulations are performed to verify our scheme. It can be found that from the 2D PIC simulations, by adding the matched auxiliary laser from the other side of the foil, the curve of the foil can be effectively counteracted. Two counterpropagating transversely circularly polarized $(C P)$ Gaussian lasers with normalized amplitudes $a_{1}=120$ and $a_{2}=30$ respectively impinge on the foil target at the meantime, a $1.3 \mathrm{GeV}$ monoenergetic proton beam with high collimation is obtained finally. Therefore, an efficient RPA of a common flat foil can be realized and a high quality proton beam can be generated finally. By varying the different parameters of the auxiliary laser, the effects of different parameters of the auxiliary laser in our scheme are investigated, and it is found that all the parameters of the auxiliary laser, including the intensity, the waist radius, and the pulse width, have influences on the quality of the accelerated ion beam. In order to generate the high quality ion beam through our scheme, the parameters of the main and auxiliary lasers should satisfy the matching condition, and these two lasers are supposed to impinge on the foil at the meantime. It should be noted that this paper has been primarily concerned with a high quality ion acceleration scheme through the relatively uniform RPA process of a flat foil target, by suppressing the target curve. The target curve can also be helpful in some other ion acceleration schemes. Compared with the previous researches for resolving the target curve of the RPA, the scheme we proposed, in which the normal Gaussian lasers and a common flat foil target are used, could relatively reduce the technology requirement for the high quality ion acceleration in the RPA experiment. In consequence, the high quality ion beam can be generated through this new scheme in a more likely promising way, which may have many significant applications, i.e., tabletop accelerator, tumor therapy and inertial confinement fusion. 


\section{ACKNOWLEDGMENTS}

This work is supported by the National Natural Science Foundation of China (NNSFC) (Grants No. 11365020, No. 11765017, No. 11475026, No. 11565022, and No. 11547304), the Science and Technology Program of Gansu Province of China (Grant No. 1606RJZA090), the Fundamental Research Funds for the Higher Education Institutions of Gansu Province of China (2012), and the Foundation of Northwest Normal University (Grants No. NWNU-LKQN-14-9 and No. NWNU-LKQN-16-3). B. S. Xie is also partially supported by the Fundamental Research Funds for the Central Universities (FRFCU).

[1] J. W. Shearer, J. Garrison, J. Wong, and J. E. Swain, Pair Production by Relativistic Electrons from an Intense Laser Focus, Phys. Rev. A 8, 1582 (1973).

[2] E. Fourkal, B. Shahine, M. Ding, J. S. Li, T. Tajima, and C. M. Ma, Particle in cell simulation of laser-accelerated proton beams for radiation therapy, Med. Phys. 29, 2788 (2002).

[3] M. Roth, T. E. Cowan, M. H. Key, S. P. Hatchett, C. Brown, W. Fountain, J. Johnson, D. M. Pennington, R. A. Snavely, S. C. Wilks, K. Yasuike, H. Ruhl, F. Pegoraro, S. V. Bulanov, E. M. Campbell, M. D. Perry, and H. Powell, Fast Ignition by Intense Laser-Accelerated Proton Beams, Phys. Rev. Lett. 86, 436 (2001).

[4] E. Esarey, C. B. Schroeder, and W. P. Leemans, Physics of laser-driven plasma-based electron accelerators, Rev. Mod. Phys. 81, 1229 (2009).

[5] D. W. Forslund and C. R. Shonk, Formation and Structure of Electrostatic Collisionless Shocks, Phys. Rev. Lett. 25, 1699 (1970).

[6] D. W. Forslund and J. P. Freidberg, Theory of Laminar Collisionless Shocks, Phys. Rev. Lett. 27, 1189 (1971).

[7] J. Denavit, Absorption of high-intensity subpicosecond lasers on solid density targets, Phys. Rev. Lett. 69, 3052 (1992).

[8] L. O. Silva, M. Marti, J. R. Davies, R. A. Fonseca, C. Ren, F. S. Tsung, and W. B. Mori, Proton Shock Acceleration in Laser-Plasma Interactions, Phys. Rev. Lett. 92, 015002 (2004).

[9] H. Habara et al., Ion acceleration from the shock front induced by hole boring in ultraintense laser-plasma interactions, Phys. Rev. E 70, 046414 (2004).

[10] A. Maksimchuk, S. Gu, K. Flippo, D. Umstadter, and V. Y. Bychenkov, Forward Ion Acceleration in Thin Films Driven by a High-Intensity Laser, Phys. Rev. Lett. 84, 4108 (2000).

[11] R. A. Snavely, M. H. Key, S. P. Hatchett, T. E. Cowan, M. Roth, T. W. Phillips, M. A. Stoyer, E. A. Henry, T. C. Sangster, M.S. Singh, S. C. Wilks, A. MacKinnon, A. Offenberger, D. M. Pennington, K. Yasuike, A. B. Langdon, B. F. Lasinski, J. Johnson, M. D. Perry, and E. M. Campbell, Intense High-Energy Proton Beams from Petawatt-Laser Irradiation of Solids, Phys. Rev. Lett. 85, 2945 (2000).

[12] S. C. Wilks, A. B. Langdon, T. E. Cowan, M. Roth, M. Singh, S. Hatchett, M. H. Key, D. Pennington,
A. MacKinnon, and R. A. Snavely, Energetic proton generation in ultra-intense laser-solid interactions, Phys. Plasmas 8, 542 (2001).

[13] J. Schreiber, F. Bell, F. Grüner, U. Schramm, M. Geissler, M. Schnürer, S. Ter-Avetisyan, B. M. Hegelich, J. Cobble, E. Brambrink, J. Fuchs, P. Audebert, and D. Habs, Analytical Model for Ion Acceleration by High-Intensity Laser Pulses, Phys. Rev. Lett. 97, 045005 (2006).

[14] G. A. Askar'yan, S. V. Bulanov, F. Pagoraro, and A. M. Pukhov, Magnetic interaction of self-focusing channels and fluxes of electromagnetic radiation: their coalescence, the accumulation of energy, and the effect of external magnetic fields on them, JETP Lett. 60, 251 (1994).

[15] S. V. Bulanov, M. Lontano, T. Z. Esirkepov, F. Pegoraro, and A. M. Pukhov, Electron Vortices Produced by Ultraintense Laser Pulses, Phys. Rev. Lett. 76, 3562 (1996).

[16] G. Mourou, Z. Chang, A. Maksimchuk, J. Nees, S. V. Bulanov, V. Y. Bychenkov, T. Z. Esirkepov, N. M. Naumova, F. Pegoraro, and H. Ruhl, On the design of experiments for the study of relativistic nonlinear optics in the limit of single-cycle pulse duration and single-wavelength spot size, Plasma Phys. Rep. 28, 12 (2002).

[17] S. V. Bulanov and T. Z. Esirkepov, Comment on "Collimated Multi-MeV Ion Beams from High-Intensity Laser Interactions with Underdense Plasma", Phys. Rev. Lett. 98, 049503 (2007).

[18] A. Sharma and A. Andreev, Effective laser driven proton acceleration from near critical density hydrogen plasma, Laser Part. Beams 34, 219 (2016).

[19] T. Esirkepov, M. Borghesi, S. V. Bulanov, G. Mourou, and T. Tajima, Highly Efficient Relativistic-Ion Generation in the Laser-Piston Regime, Phys. Rev. Lett. 92, 175003 (2004).

[20] B. F. Shen and Z.Z. Xu, Transparency of an overdense plasma layer, Phys. Rev. E 64, 056406 (2001).

[21] A. Macchi, F. Cattani, T. V. Liseykina, and F. Cornolti, Laser Acceleration of Ion Bunches at the Front Surface of Overdense Plasmas, Phys. Rev. Lett. 94, 165003 (2005).

[22] F. Pegoraro and S. V. Bulanov, Photon Bubbles and Ion Acceleration in a Plasma Dominated by the Radiation Pressure of an Electromagnetic Pulse, Phys. Rev. Lett. 99, 065002 (2007).

[23] X. M. Zhang, B. F. Shen, X. M. Li, Z. Y. Jin, F. C. Wang, and $\mathrm{M}$. Wen, Efficient $\mathrm{GeV}$ ion generation by ultraintense circularly polarized laser pulse, Phys. Plasmas 14, 123108 (2007).

[24] M. Chen, A. Pukhov, Z. M. Sheng, and X. Q. Yan, Laser mode effects on the ion acceleration during circularly polarized laser pulse interaction with foil targets, Phys. Plasmas 15, 113103 (2008).

[25] A. P. L. Robinson, M. Zepf, S. Kar, R. G. Evans, and C. Bellei, Radiation pressure acceleration of thin foils with circularly polarized laser pulses, New J. Phys. 10, 013021 (2008).

[26] O. Klimo, J. Psikal, J. Limpouch, and V. T. Tikhonchuk, Monoenergetic ion beams from ultrathin foils irradiated by ultrahigh-contrast circularly polarized laser pulses, Phys. Rev. ST Accel. Beams 11, 031301 (2008).

[27] X. Q. Yan, C. Lin, Z. M. Sheng, Z. Y. Guo, B. C. Liu, Y. R. Lu, J. X. Fang, and J. E. Chen, Generating High-Current 
Monoenergetic Proton Beams by a Circularly Polarized Laser Pulse in the Phase-Stable Acceleration Regime, Phys. Rev. Lett. 100, 135003 (2008).

[28] M. Chen, A. Pukhov, T. P. Yu, and Z. M. Sheng, Enhanced Collimated GeV Monoenergetic Ion Acceleration from a Shaped Foil Target Irradiated by a Circularly Polarized Laser PulsePhys. Rev. Lett. 103, 024801 (2009).

[29] A. Macchi, S. Veghini, and F. Pegoraro, "Light Sail" Acceleration Reexamined, Phys. Rev. Lett. 103, 085003 (2009).

[30] A. Henig, S. Steinke, M. Schnürer, T. Sokollik, R. Hörlein, D. Kiefer, D. Jung, J. Schreiber, B. M. Hegelich, X. Q. Yan, J. M. ter Vehn, T. Tajima, P. V. Nickles, W. Sandner, and D. Habs, Radiation-Pressure Acceleration of Ion Beams Driven by Circularly Polarized Laser Pulses, Phys. Rev. Lett. 103, 245003 (2009).

[31] A. P. L. Robinson, D. H. Kwon, and K. Lancaster, Holeboring radiation pressure acceleration with two ion species, Plasma Phys. Controlled Fusion 51, 095006 (2009).

[32] A. P. L. Robinson, P. Gibbon, M. Zepf, S. Kar, R. G. Evans, and C. Bellei, Relativistically correct hole-boring and ion acceleration by circularly polarized laser pulses, Plasma Phys. Controlled Fusion 51, 024004 (2009).

[33] V. K. Tripathi, C. S. Liu, X. Shao, B. Eliasson, and R. Z. Sagdeev, Laser acceleration of monoenergetic protons in a self-organized double layer from thin foil, Plasma Phys. Controlled Fusion 51, 024014 (2009).

[34] T. P. Yu, A. Pukhov, G. Shvets, and M. Chen, Stable LaserDriven Proton Beam Acceleration from a Two-Ion-Species Ultrathin Foil, Phys. Rev. Lett. 105, 065002 (2010).

[35] B. Qiao, M. Zepf, M. Borghesi, B. Dromey, M. Geissler, A. Karmakar, and P. Gibbon, Radiation-Pressure Acceleration of Ion Beams from Nanofoil Targets: The Leaky Light-Sail Regime, Phys. Rev. Lett. 105, 155002 (2010).

[36] X. R. Hong, B. S. Xie, S. Zhang, H. C. Wu, A. Amidula, X. Y. Zhao, and M. P. Liu, High quality ion acceleration from a double-layer target dominated by the radiation pressure of a transversely Gaussian laser pulse, Phys. Plasmas 17, 103107 (2010).

[37] A. Macchi, M. Borghesi, and M. Passoni, Ion acceleration by superintense laser-plasma interaction, Rev. Mod. Phys. 85, 751 (2013).

[38] J. H. Bin, W. J. Ma, H. Y. Wang, M. J. V. Streeter, C. Kreuzer, D. Kiefer, M. Yeung, S. Cousens, P. S. Foster, B. Dromey, X. Q. Yan, R. Ramis, J. M. ter Vehn, M. Zepf, and J. Schreiber, Ion Acceleration Using Relativistic Pulse Shaping in Near-Critical-Density Plasmas, Phys. Rev. Lett. 115, 064801 (2015).

[39] S. S. Bulanov, E. Esarey, C. B. Schroeder, S. V. Bulanov, T. Z. Esirkepov, M. Kando, F. Pegoraro, and W.P. Leemans, Enhancement of Maximum Attainable Ion Energy in the Radiation Pressure Acceleration Regime Using a Guiding Structure, Phys. Rev. Lett. 114, 105003 (2015).

[40] C. Lv, B. S. Xie, F. Wan, Y. J. Hou, M. R. Jia, H. B. Sang, X.R. Hong, and S.B. Liu, Enhanced laser radiation pressure acceleration of protons with a gold cone-capillary, Phys. Plasmas 24, 033122 (2017).

[41] X. R. Hong, W. J. Zhou, B. S. Xie, Y. Yang, L. Wang, J. M. Tian, R. A. Tang, and W. S. Duan, Generation of high quality ion beams through the stable radiation pressure acceleration of the near critical density target, Chin. Phys. B 26, 065203 (2017).

[42] Y. Wan, C. H. Pai, C. J. Zhang, F. Li, Y. P. Wu, J. F. Hua, W. Lu, Y. Q. Gu, L. O. Silva, C. Joshi, and W. B. Mori, Physical Mechanism of the Transverse Instability in Radiation Pressure Ion Acceleration, Phys. Rev. Lett. 117, 234801 (2016).

[43] C. Nieter and J. R. Cary, VORPAL: a versatile plasma simulation code, J. Comput. Phys. 196, 448 (2004).

[44] P. Gibbon, Short Pulse Laser Interaction with Matter: An Introduction (Imperial College Press, London, 2005). 